\title{
EVALUACIÓN DE PARAMETROS FISIOLÓGICOS Y BIOLÓGICOS SELECCIONADOS PARA VALORAR LA CONDICIÓN DEL ALOJAMIENTO ENRIQUECIDO EN RATAS Y RATONES PRODUCIDOS EN INDICASAT AIP
}

\author{
De Jesús ${ }^{1}$, Rosa $^{1}$ \\ INDICASAT AIP \\ Panamá, Panamá \\ https://orcid.org/0000-0001-9670-291X \\ Madrid², Alanna2 \\ INDICASAT AIP \\ Panamá, Panamá \\ amadrid@indicasat.org.pa \\ Rivera ${ }^{3}$, René ${ }^{3}$ \\ INDICASAT AIP \\ Panamá, Panamá \\ rrivera@indicasat.org.pa \\ Herrera ${ }^{4}$, Lizzi $^{4}$ \\ INDICASAT AIP \\ Panamá, Panamá \\ Iherrera@indicasat.org.pa
}

\begin{abstract}
The enrichment environmental actually in Laboratory animal research could be considered a new paradigm. The objective of these assay was to observe if the physiology and biologics parameters can be affected when the laboratory animals are maintained in an enrichment environment or in not an enrichment environment, affecting the reproductivity of the results. Assay used ten (10) mice (C57BL/ 6 y BALB/C), ( $n=5 /$ experimental group) and eight (8) rats Sprague Dawley ( $n=4 /$ experimental group), all was female. The animals were weaned and they were housed two group, one with enviroment enrichment and other without enrichment. The enrichment environment counted with two (2) Polyvinylchloride tubes and paper towel. Evaluated weekly weight, food and water consumer during eight
\end{abstract}


(8) weeks when were realized hemoglobine, hematocrite and glucemia test, the blood sample was obtained across retroorbital via, with the animal previous anesthetized. The results present that there is no significant difference in relation to biologics parameters of food and water consumption for the animals housed in neither of the two environments with enrichment and without enrichment. Significant difference was found in the hemoglobin value ( $p>0.05)$, the value was higher in the animals housed without enrichment environmental. The hematocrit and glucose values were higher of the animals housed in enrichment environmental. Environmental enrichment played an important role in the valores of physiological tests, such as hematological and biochemical blood tests, which can lead to the non-reproducibility of the results of an in vivo investigation.

Keywords: enriched environment, rats, mice, blood test.

\section{Resumen}

El enriquecimiento ambiental actualmente puede ser considerado como un nuevo paradigma en la investigación con los animales de laboratorio. El objetivo del presente ensayo fue el de observar si algunos parámetros biológicos y fisiológicos podían ser afectados cuando los animales que son usados para la investigación son alojados en ambientes enriquecidos y no enriquecidos. En el ensayo se usaron diez (10) ratones C57BL/6 y BALB/C, usando un $\mathrm{n}=5$ ratones/grupo experimental, y ocho (8) ratas Sprague Dawley (con un $\mathrm{n}=4$ ratas/grupo experimental), todos los animales ensayos fueron hembras. Los animales al destete se dividieron en dos grupos uno se alojó en una caja con enriquecimiento y el otro en una caja sin enriquecimiento. El enriquecimiento consistió en dos (2) tubos de polivinil cloruro y una hoja de papel toalla. Se evaluó semanalmente el peso, el consumo de alimento y agua y a las ocho semanas se realizaron pruebas de hemoglobina, hematocrito y glicemia, de muestra sanguínea obtenida por vía retroorbital en los animales anestesiados. Los resultados no presentaron diferencias significativas en la valoración de los parámetros biológicos. Para los valores de hemoglobina se observaron diferencias significativas para los animales alojados sin enriquecimiento y se observaron diferencias significativas para los valores de hematocrito y glucosa para los animales alojados en el ambiente con enriquecimiento. Se concluye que el enriquecimiento ambiental juega un papel importante en los valores relacionados a parámetros fisiológicos, tales como hemoglobina, hematocrito y glucosa, lo cual puede conducir a la no reproducibilidad de los resultados experimentales.

Palabras claves: enriquecimiento ambiental, ratas, ratones, pruebas sanguíneas. 


\section{INTRODUCCIÓN}

Se considera que el enriquecimiento ambiental es útil para una amplia gama de experimentos que estudian los efectos del medio ambiente en los ratones. Se ha reportado, igualmente la influencia de este en datos de experimentos tanto de cáncer como de metabolismo y el efecto sobre el crecimiento, el peso de los órganos y en parámetros fisiológicos [1,2,3].

En el mantenimiento de los animales usados en la investigación in vivo se establece como un nuevo paradigma el enriquecimiento ambiental como un motivador del bienestar animal.

Asumiendo el concepto de bienestar animal (en inglés traducido como Welfare) según la Organización Mundial de Sanidad Animal (OIE) expuesto en el documento: Código Sanitario para los Animales Terrestres [4] en el capítulo 7.1 denominado Introducción a las recomendaciones para el bienestar de los animales: Artículo 7.1.1: "Se define como el modo en que un animal afronta las condiciones de su entorno. Un animal está en buenas condiciones de bienestar si (según indican pruebas científicas) está sano, cómodo, bien alimentado, en seguridad, puede expresar formas innatas de comportamiento y si no padece sensaciones desagradables de dolor, miedo o desasosiego. El enriquecimiento ambiental es una técnica de alojamiento de los animales compuesto por un mayor espacio, la actividad física, y las interacciones sociales entre otras, que a su vez aumenta lo sensorial, lo cognitivo, la actividad motora y el estímulo social de estos. La estrategia del enriquecimiento ambiental, ofrece retos leves y breves que inducen una respuesta adaptativa beneficiosa, benigna, y saludable, conocido como eustress, en oposición a un entorno hostil más aversivo o angustia no adaptativa Al promover eustress y limitar la angustia perjudicial, los ratones muestran una variedad de fenotipos favorables, al igual que cuando los animales se encuentran en condición de estrés, ambos comportamientos pueden ser valorados.

Es importante determinar el efecto que realmente el enriquecimiento ambiental tiene sobre los resultados de una experiencia antes de introducirlo rutinariamente como parte de la producción en un bioterio, de forma que, si se plantea mantener a los animales bajo condiciones de enriquecimiento ambiental, el investigador pueda estar al tanto si este beneficia o no sus datos experimentales y en consecuencia realizar conclusiones adecuadas de sus ensayos, y decidir si lo le es conveniente para los animales usados en sus investigaciones o sino lo es, y justificarlo ante el CICUA en el momento de solicitar el aval al desarrollo del mismo.

En este ensayo se valora si los animales alojados en ambientes no enriquecidos ajustan de forma diferente sus parámetros fisiológicos a como lo hacen los alojados en ambientes enriquecidos, lo que puede traducirse en diferencias de los valores 
de parámetros biológicos y fisiológicos medidos como datos en las investigaciones.

\section{MÉTODO}

\section{A. ANIMALES USADOS}

Se usaron diez ratones ( $C 57 B L / 6$ y $B A L B / C),(n=5 / g r u p o)$ y ocho ratas Sprague Dawley ( $n=4 /$ grupo), hembras. Al destete se establecieron los grupos: con enriquecimiento (CE), y sin enriquecimiento (SE).

\section{B. PARÁMETROS EVALUADOS}

Se evaluó el peso semanal, consumo de alimento y agua. A las 8 semanas se realizaron pruebas de hemoglobina, hematocrito y glicemia.

\section{DISEÑO DESARROLLADO}

Los animales se alojaron en el Bioterio del INDICASAT AIP a una temperatura de 18 ${ }^{\circ} \mathrm{C}$, con ciclos de luz oscuridad de $12 \mathrm{~h}$ luz/12 h oscuridad. Alojados en instalaciones de alta barrera biológica (Jackson Laboratory, 2021), tales como: ducha del personal al entrar, uniforme de trabajo, uso de mascarilla, uso de guantes, esterilización de viruta y alimento $\left(121^{\circ} \mathrm{C} / 15 \mathrm{~min}\right)$. Con promedios entre 115 - 119 luxes de intensidad de luz, promediado entre tres medidas en distintos puntos de la caja.

A los dos grupos experimentales, al término de las 8 semanas se les realizó una toma de muestra de sangre vía retroorbital previa sedación con isofluorano (100\% en cámara de vidrio), comprobada por ninguna respuesta a estímulos podales de las cuatro extremidades. El procedimiento se aplicó usando un capilar de $70 \mathrm{~mm}( \pm 0,05)$ de $70 \mu \mathrm{L}$, para realizar prueba de glucosa, hemoglobina y hematocrito. La glucosa se midió mediante el kit de diagnóstico (Marca Human - Método GOD-PAP. Prueba enzimática colorimétrica por glucosa. Alemania), esta se realizó en un equipo semiautomático BTS 350 (BioSystems. España), el mismo equipo fue usado para medir la concentración de hemoglobina usando el kit de diagnóstico Hemoglobin liquicolor (marca Human-Método de la cianmetahemoglobina). El hematocrito se realizó usando capilares heparinizados, centrifugándolos en Microcentrifuga. Luego de la toma de muestra los animales se les aplicó eutanasia devolviéndolos a la cámara de anestesia.

\section{ANÁLISIS ESTADÍSTICO REALIZADO}

Para observar la normalidad de los resultados se usó la prueba Kolmogorov-Smirrnov, con un $p>0,05$. Los resultados se valoraron mediante un análisis de varianza ANOVA se usó el programa estadístico Statixtic 2010.

Este ensayo se realizó en la Unidad de Producción y Experimentación Animal de INDICASAT AIP, con el aval del CICUA N²0-002. 


\section{RESUltados}

No se encontraron diferencias significativas con respecto a los parámetros biológicos medidos, entre los animales que se alojaron en condiciones de enriquecimiento ambiental y los que se alojaron en condiciones de alojamiento sin enriquecimiento. Los animales que se alojaron en ambiente sin enriquecimiento presentaron un mayor consumo de agua y de alimento que los alojados en ambientes sin enriquecimiento, sin embargo, no fue significativo.

Las Figuras 1, 2 y 3, presentan la tendencia del crecimiento del grupo de ratas hembras Sprague Dawley y de los ratones hembras BALB/c y C57BL/6, respectivamente, alojados en un ambiente enriquecido y en un ambiente no enriquecido. La tendencia no presentó diferencias significativas entre grupos para un nivel de significancia de $p<0.05 ; p=0.38$, la tendencia observada para el grupo de las hembras se observó igualmente para el grupo de los machos tanto de las ratas como de los ratones machos. En las primeras 5 semanas de crecimiento los animales alojados en el ambiente sin enriquecimiento presentaron un mayor promedio de crecimiento que fue similar luego de esta semana al de los animales con enriquecimiento.
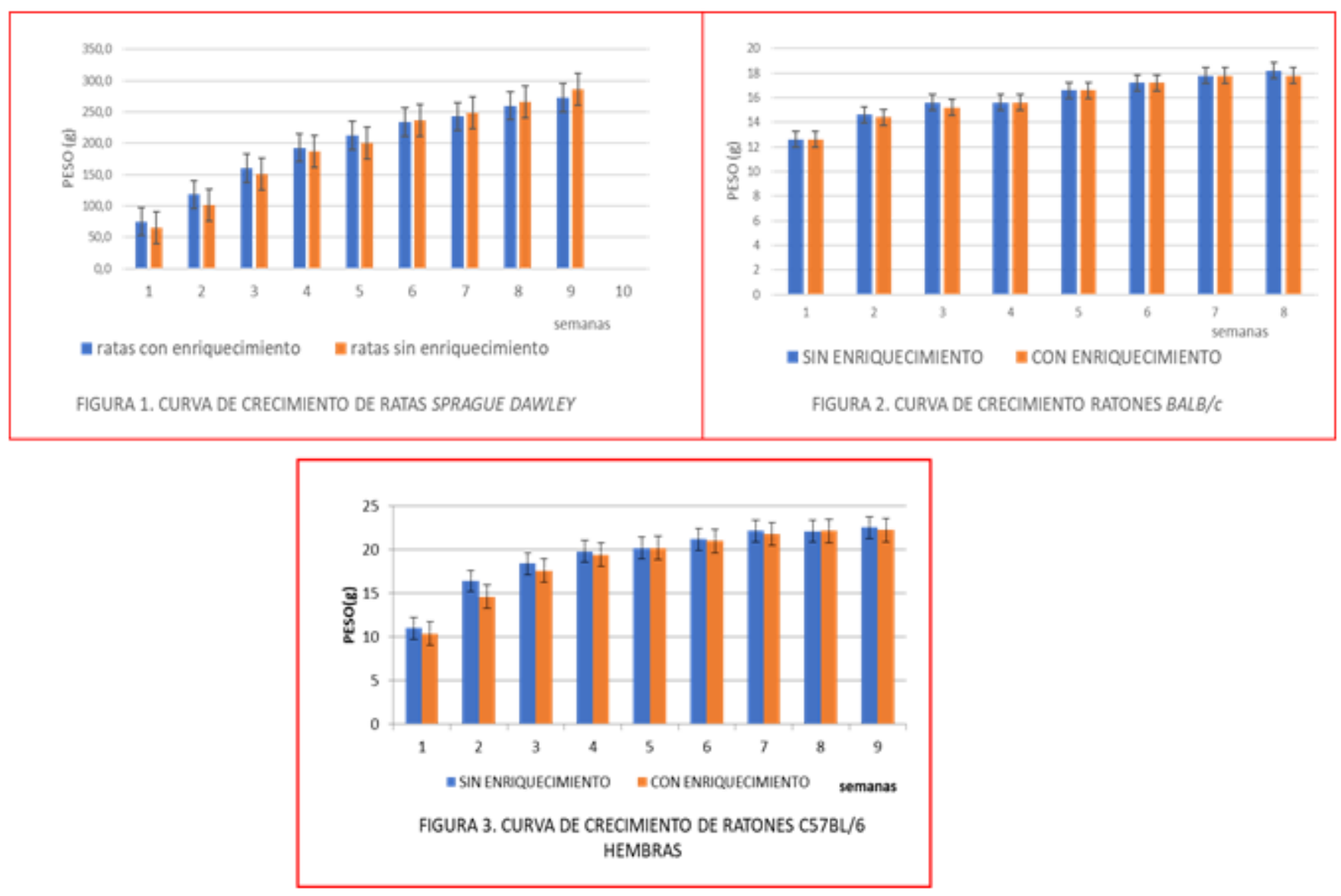
En la Tabla 1, se pueden observar los valores de los parámetros bioquímicos sanguíneos: hemoglobina, hematocrito y glucosa de los ratones y ratas del ensayo

\begin{tabular}{|c|c|c|c|c|c|c|}
\hline & \multicolumn{2}{|c|}{ C57BL/6 } & \multicolumn{2}{c|}{ BALB/C } & \multicolumn{2}{c|}{ SPRAGUE DAWLEY } \\
\hline & $\mathrm{SE}$ & $\mathrm{CE}$ & $\mathrm{SE}$ & $\mathrm{CE}$ & $\mathrm{SE}$ & $\mathrm{CE}$ \\
\hline $\mathrm{Hb}$ & $16,98 \pm 2,6$ & $14,56 \pm 3,0$ & $16 ., 4 \pm 1,9$ & $15,66 \pm 1,5$ & $15,3 \pm 2,0$ & $13,5 \pm 1,2$ \\
\hline Glucosa & $159,2 \pm 1, .8$ & $200 \pm 15,2$ & $186,8 \pm 68,1$ & $318,4 \pm 31,48$ & $124,5 \pm 12,9$ & $120,2 \pm 18,8$ \\
\hline Hematocrito & $48 \pm 6,2$ & $50,8 \pm 4,1$ & $50,8 \pm 3,0$ & $53,2 \pm 1,6$ & $45,2 \pm 2,2$ & $47 \pm 1,7$ \\
\hline
\end{tabular}

Se puede observar en la tabla que los valores de hemoglobina fueron más altos en los animales alojados sin enriquecimiento. Los valores del hematocrtio y la glucosa presentaron promedios mayores en los animales alojados en ambientes con enriquecimiento ambos presentaron diferencias significativas para un $p>0,05 ; p=0,015$.

\section{CONCLUSIONES}

El ensayo desarrollado se realizó en dos especies distintas y en dos líneas de ratones genéticamente diferentes encontrando que los PARÁMETROS BIOLÓGICOS no presentaron diferencias significativas en relación a los diferentes alojamientos usados: con enriquecimiento y sin enriquecimiento. Sin embargo, los parámetros hematológicos (hemoglobina y hematocrito) y el bioquímico medido, presentaron diferencias significativas que pueden estar relacionadas con la condición del enriquecimiento del ambiente de los animales. Por lo que se concluye que los resultados de una investigación pueden no ser reproducibles cuando se consideran estos parámetros para concluir los resultados experimentales. los investigadores deben reportar esta condición en el aparte de materiales y métodos para lograr la reproducibilidad de sus resultados.

\section{Referencias}

[1] L. Cao, E. Choi, X. Liu, A. Martin, Ch. Wang, X. Xu, M. During. White to brown fat phenotypic switch induced by genetic and environmental activation of a hypothalamicadipocyte axis. Cell Metab. 14(3). pp.324-338. 2011.

[2] L. Cao, X. Liu, E. Lin, Ch. Wang, E. Choi, V. Riban, B. Lin, M. During. Environmental and genetic activation of a brain-adipocyte BDNF/ leptin axis causes cancer remission and inhibition. Cell. 142(1). pp. 52-64. 2010.

[3] P. Tsai, U. Pachowsky, H. Stelzer, H. Hackbarth. Impact of environmental enrichment in mice. 1: Effect of housing conditions on body weight, organ weights and haematology indifferent strains. Laboratory Animals Ltd. Laboratory Animals. 36.pp. 411-419. 2002. 
[4] https://www.oie.int/fileadmin/Home/esp/Health_standards/tahc/current/es_sommaire.html Consultada: Enero 2021.

\section{Autorización y Licencia CC}

Los autores autorizan a APANAC XVIII a publicar el artículo en las actas de la conferencia en Acceso Abierto (Open Access) en diversos formatos digitales (PDF, HTML, EPUB) e integrarlos en diversas plataformas online como repositorios y bases de datos bajo la licencia CC:

Attribution-NonCommercial-ShareAlike 4.0 International (CC BY-NC-SA 4.0) https://creativecommons.orq/ licenses/by-nc-sa/4.0/.

Ni APANAC XVIII ni los editores son responsables ni del contenido ni de las implicaciones de lo expresado en el artículo. 